\title{
Extragalactic globular clusters in the near infrared
}

\section{A comparison between M87 and NGC 4478 ${ }^{\star}$}

\author{
M. Kissler-Patig ${ }^{1}$, J. P. Brodie ${ }^{2}$, and D. Minniti ${ }^{3}$ \\ 1 European Southern Observatory, Karl-Schwarzschild-Str. 2, 85748 Garching, Germany \\ e-mail: mkissler@eso.org \\ 2 UCO/Lick observatory, University of California, Santa Cruz, CA 95064, USA \\ e-mail: brodie@ucolick.org \\ 3 Departamento de Astronomía y Astrofísica, P. Universidad Católica, Casilla 104, Santiago 22, Chile \\ e-mail: dante@astro.puc.cl
}

Received 2 January 2002 / Accepted 4 June 2002

\begin{abstract}
We compare optical and near infrared colours of globular clusters in M87, the central giant elliptical in Virgo, and NGC 4478, an intermediate luminosity galaxy in Virgo, close in projection to M87. Combining $V$ and $I$ photometry obtained with the WFPC 2 on HST and $K_{\mathrm{s}}$ photometry obtained with the NIRC on Keck 1, we find the broad range in colour and previously detected bi-modality in M87. We confirm that NGC 4478 only hosts a blue sub-population of globular clusters and now show that these clusters' $V-I$ and $V-K$ colours are very similar to those of the halo globular clusters in Milky Way and M31. Most likely, a metal-rich sub-population never formed around this galaxy (rather than having formed and been destroyed later), probably because its metal-rich gas was stripped during its passage through the centre of the Virgo cluster.

The $V-I, V-K$ colours are close to the predicted colours from SSP models for old populations. However, M87 hosts a few red clusters that are best explained by intermediate ages (a few Gyr). Generally, there is evidence that the red, metal-rich sub-population has a complex colour structure and is itself composed of clusters spanning a large metallicity and, potentially, age range. This contrasts with the blue, metal-poor population which appears very homogeneous in all galaxies observed so far.
\end{abstract}

Key words. galaxies clusters: general - galaxies: star clusters - galaxies: individual: M87, NGC 4478

\section{Introduction}

Globular cluster systems are now established as useful tools for studying the formation and evolution of their host galaxies (see recent reviews by Ashman \& Zepf 1998; Kissler-Patig 2000; Harris 2001). One of the most interesting aspects of this approach is the composite nature of most globular cluster systems. Many systems of early-type galaxies appear to be composed of two or more distinct sub-populations of globular clusters (Zepf \& Ashman 1993; see also recently Gebhardt \& Kissler-Patig 1999; Larsen et al. 2001; Kundu \& Whitmore 2001a,b). A number of groups have concentrated on studying the properties of the individual globular cluster sub-populations in a galaxy (e.g. Kissler-Patig 2002 for a recent overview, or above reviews) with the goal of revealing the origins of the

Send offprint requests to: M. Kissler-Patig,

e-mail: mkissler@eso.org

* Data presented herein were obtained at the W.M. Keck Observatory, which is operated as a scientific partnership among the California Institute of Technology, the University of California and the National Aeronautics and Space Administration. The Observatory was made possible by the generous financial support of the W.M. Keck Foundation. sub-populations and thus shedding light on the host galaxy formation and evolution. To summarize the results so far, the blue and the red globular cluster sub-populations differ significantly in their spatial distributions, mean sizes, metallicities and kinematics while, in contrast, they appear to have similar old ages within the large (2-4 Gyr) errors. All this indicates that there must have been significant differences in the origins of the two main sub-populations (e.g. Burgarella et al. 2001 and references therein).

The keys to studying differences in sub-population properties is their unambiguous identification, and a reliable association of the individual clusters with the different subpopulations. The identification of sub-populations started in the Milky Way with the landmark paper of Zinn (1985), but another decade elapsed before they were revealed in early-type galaxies (Zepf \& Ashman 1993; Lee \& Geisler 1993, and above references for the long lists of following studies). Observationally, the sub-populations are primarily identified in the metallicity distribution of the entire system. While for nearby galaxies (including the Milky Way) the metallicities are determined spectroscopically, we rely on photometric studies and colourto-metallicity conversions for the vast majority of (further) early-type galaxies. 
This immediately prompts the questions: how metallicity sensitive is the photometry? Especially given the agemetallicity degeneracy of broad band colours (e.g. Worthey 1994). Most studies (in particular the ones done with the WFPC2) used broad band colours in the Johnson-Cousins systems or equivalent which are poorly suited to disentangling sub-populations.

This motivated our choice to work with a combination of optical and near-infrared colours. The long wavelength baseline provides the most metallicity sensitivity, allowing the subpopulations to be distinguished by their colours even if they are very close in metallicity. Historically, the first near-infrared measurements of extragalactic globular clusters were carried out in M31 (Frogel et al. 1980) and the Large Magellanic Cloud (Persson et al. 1983). Technical advancement both in the near-infrared arrays, as well as in telescope sizes allows us now to study globular clusters beyond the Local Group with high photometric accuracy. The first studies beyond the Local Group focused on young/intermediate age star clusters, whose AGB stars contribute significantly to the $K$ magnitude (see Minniti et al. 1996 and Rejkuba 2001 for studies in Cen A, Maraston et al. 2001 for studies in NGC 7252, Goudfrooij et al. 2001 for studies of NGC 1316).

Our larger project aims at studying a dozen galaxies of different luminosities and in different environments. In the first paper of the series, we study the Virgo Galaxies Messier 87 and NGC 4478. Their properties are summarized in Table 1. M87 is the central giant elliptical in Virgo while NGC 4478 is an intermediate luminosity galaxy in Virgo, close in projection to M87.

Our near-infrared data (NIR) and the combination with optical data from WFPC2 on HST, are described in Sect. 2. The $V, I, K$ colour distributions presented in Sect. 3. Our goals are threefold: first, we will study the colour/metallicity distribution of the globular clusters systems, taking advantage of the higher metallicity resolution afforded by the NIR, to shed light on the sub-populations origins. In particular, NGC 4478 has been reported as having hardly any metal-rich globular clusters (Neilsen et al. 1997), which is unusual for a galaxy of that luminosity. Second, we will compare the optical - near-infrared colours with existing population synthesis models and data from the Milky Way and M31. This is valuable because NIR data of "clean" single stellar populations with high metallicities, suitable for comparison with models, are sparse. Finally, for M87, we will look for sub-structure within the red subpopulation and probe the metallicity dispersion of the metalrich clusters. A summary of our findings and conclusions are given in Sect. 5.

\section{Observations and reductions}

\subsection{Near infrared photometry}

The near infrared photometry was obtained on the night of March 6th, 1998 with the Near Infrared Camera (NIRC, Matthews \& Soifer 1994) at the Keck 1 telescope. The camera is equipped with a $256 \times 256 \mathrm{InSb}$ array mounted at the $\mathrm{f} / 25$ forward Cassegrain focus of the telescope; a $30 \mu \mathrm{m}$
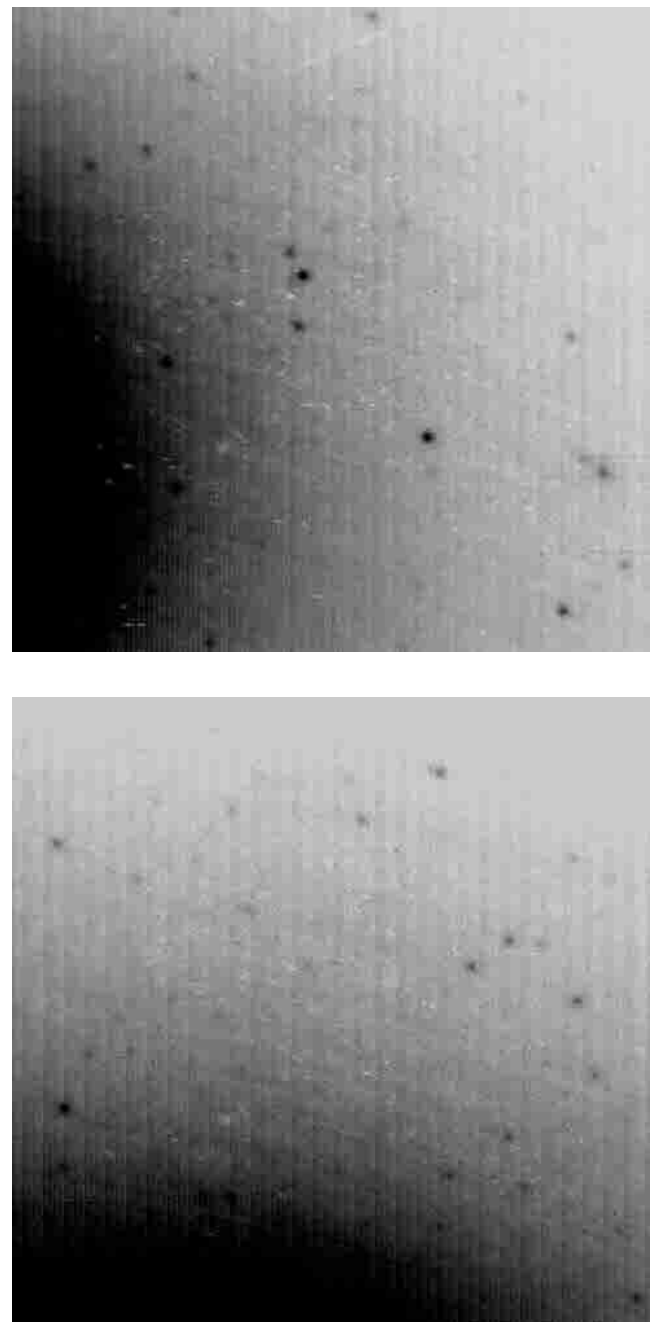

Fig. 1. Images of our two M87 fields. Upper panel: the M87 "east" field, $K_{\mathrm{s}}$ filter, $650 \mathrm{~s}$ exposure; lower panel: the M87 "west" field, $K_{\mathrm{s}}$ filter, $750 \mathrm{~s}$ exposure. Both images are $38^{\prime \prime} \times 38^{\prime \prime}$ in size, for the orientation see Fig. 8.

pixel corresponds to $0.15^{\prime \prime}$ on the sky; the total field of view is $38.4^{\prime \prime} \times 38.4^{\prime \prime}$. The observations were made using the $K_{\mathrm{s}}$ $(1.99 \mu \mathrm{m}-2.32 \mu \mathrm{m})$ filter.

We selected our NIRC fields to lie within the available WFPC2 images in order to have optical colours for all our target globular clusters. We observed two globular cluster fields in M87, adjacent (East-West), 40" south of the centre, with repeated observations of a sky field, several arcminutes away. In NGC 4478, we observed two globular cluster fields covering the centre of the galaxy (one to the North and one to the South), as well as a field $\simeq 2^{\prime}$ away that served as sky field but was still included in the WFPC2 field of view.

The total integration times for the different fields were: $750 \mathrm{~s}$ for the M87 west field, 650s for the M87 east field, and $3300 \mathrm{~s}$ for each of the three fields around NGC 4478. The seeing on all exposures, as measured from the $F W H M$ of point sources, varied between $0.4^{\prime \prime}$ and $0.6^{\prime \prime}$. A typical observing cycle of $\simeq 5$ min included the two target fields and the sky field. The sky exposures were dithered in a box pattern with a total integration time equivalent to the target exposures. A median 
Table 1. Some properties of M 87 and NGC 4478.

\begin{tabular}{lrrl}
\hline \hline Property & M 87 & NGC 4478 & Reference \\
\hline RA(2000) & 123049.7 & 123017.4 & RC3 (de Vaucouleurs et al. 1991) \\
$\operatorname{Dec}(2000)$ & +122324 & +121944 & RC3 \\
$B_{\mathrm{T} 0}$ & 9.49 & 12.21 & RC3 \\
$(B-V)_{0}$ & 0.93 & 0.88 & RC3 \\
$(m-M)$ & $31.03 \pm 0.16$ & $31.29 \pm 0.28$ & Tonry et al. (2000) \\
$\mathrm{M}_{V}$ & $-22.47 \pm 0.18$ & $-19.96 \pm 0.30$ & derived from the above data \\
\hline
\end{tabular}
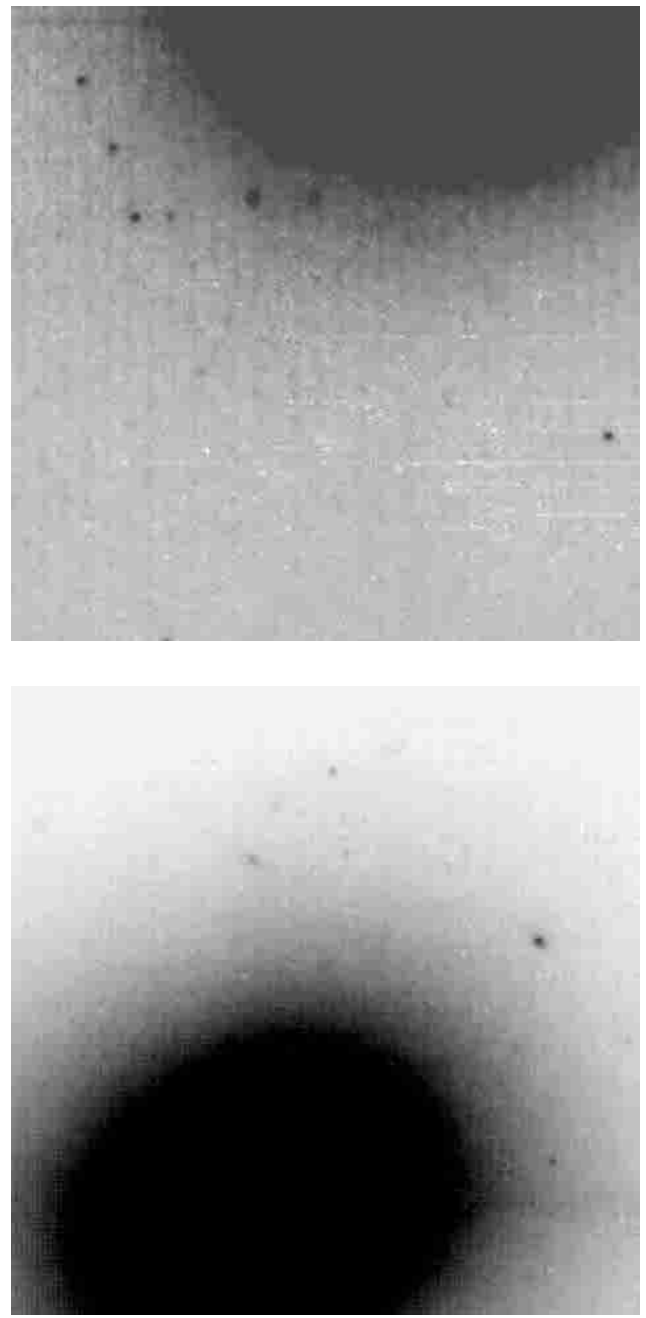

Fig. 2. Images of our two NGC 4478 fields. Upper panel: the NGC 4478 "north" field, $K_{\mathrm{s}}$ filter, $3300 \mathrm{~s}$ exposure; lower panel: the NGC 4478 "south" field, $K_{\mathrm{s}}$ filter, $3300 \mathrm{~s}$ exposure. Both images are $38^{\prime \prime} \times 38^{\prime \prime}$ in size. Same orientation on the sky as for the M87 images.

of the sky exposures was then subtracted from the target exposures to correct for dark current and sky variations. A master flat-field, obtained from all sky exposures of the night, was further used to correct for pixel to pixel variations. The target exposures thus corrected were then averaged to form a final image.

The galaxy light was efficiently removed from the frames by modelling it with a $35 \times 35$ pixel median filter. When the
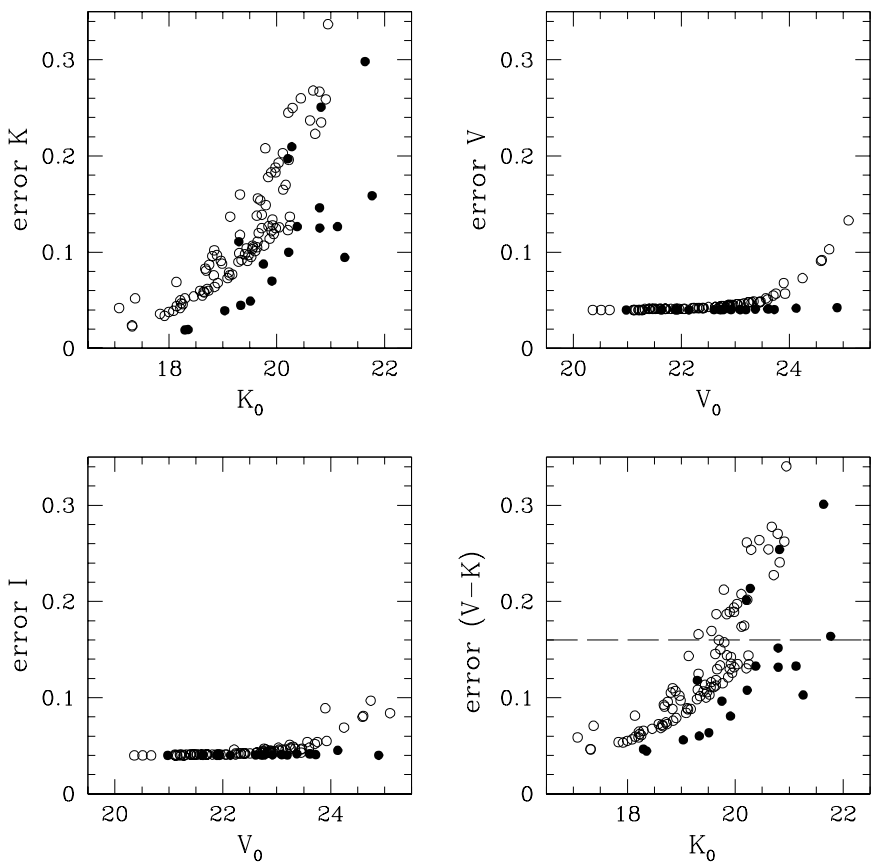

Fig. 3. Error in $K, V, I,(V-K)$ plotted as a function of $K$ and $V$ magnitude for globular cluster candidates in M87 (open circles) and NGC 4478 (solid dots). The dashed line in the lower right panel indicates our error limit $(0.16 \mathrm{mag})$ used later in the paper. Note that the error in $(V-K)$ is completely dominated by the error in $K$.

galaxy centre was located in the field, i.e. for the NGC 4478 south field, an isophotal model was computed with ISOPHOTE under IRAF. These models were subtracted from the original frames before the final object detection was started. The object finding and aperture photometry was carried out with SEXTRACTOR (Bertin \& Arnouts 1996). The fields are sufficiently un-crowded that large apertures (10-15 pixels radius) could be used and total magnitudes could be derived from a curve of growth analysis. The errors in the photometry were derived from SEXTRACTOR and are shown in Fig. 3. They are dominated by photon noise (both of the sky background and the galaxy background) as verified by measuring the bright objects on individual exposures, with different sky subtractions, and by measuring all objects on the flat-fielded and non flat-fielded images (checking for systematic errors), as well as in apertures of different sizes. Figure 3 (upper left panel) shows that for a given $K$ magnitude the error varies significantly according to the position of the object with respect to the galaxy background. Also it becomes clear that the error in $(V-K)$ is completely 
dominated by the error in $K$. Only around $K \sim 20(V \sim 23)$ does the error in $V$ start to noticeably contribute and to steepen the slope with which the error in $(V-K)$ varies with $K$ (cf. lower right panel). In addition, a group of red objects close to the galaxy center (on high background noise) mimic a "jump" in the error around $K \sim 20$. For all these reasons we chose, in what follows, to consider only clusters with $(V-K)$ errors less than 0.16 mag.

The calibration was performed with 6 standard stars (of which one was observed twice) from Persson et al.'s (1998) HST JHK standard list (SJ9108, SJ9110, SJ9134, SJ9143, SJ9149 (observed twice), and SJ9155). The standards were distributed over the night; each standard was observed in a $3 \times 3$ mosaic (medianed to compute the sky) and magnitudes were measured from each of the 9 sky-subtracted images. The 63 standard star measurements were used to derive a zero point and extinction coefficient; the colour term was assumed to be negligible. We obtained the following relation:

$m_{\text {cal }}\left(K_{\mathrm{s}}\right)=m_{\text {inst }}+24.847( \pm 0.014)-0.045( \pm 0.005) \cdot A M$,

where $m_{\text {inst }}$ is the measured magnitude of an object (normalized to $1 \mathrm{~s}$ exposure), $A M$ is the airmass, and $m_{\text {cal }}\left(K_{\mathrm{s}}\right)$ the calibrated magnitude we are seeking.

\subsection{Optical photometry}

The optical photometry was obtained from WFPC2 images of the HST Archive. For M87, we used the observations from proposal GO-5477 described in Whitmore et al. (1995). These images were obtained in the F555W $(4 \times 600 \mathrm{~s})$ and F814W $(4 \times 600 \mathrm{~s})$ filters. For NGC 4478 , we used HST parallel observations with WFPC2 described in Neilsen et al. (1997). Images were obtained in the F606W $(6 \times 2800$ s $)$ and the F814W $(5 \times 2800 s+2500 s)$.

Both Whitmore et al. (1995) and Neilsen et al. (1997) comprehensively investigated the globular cluster systems of the host galaxies in $V$ and $I$. Kundu et al. (1999) and Larsen et al. (2001) have since re-investigated the M87 data. We will come back to some of their results in the next sections. We chose to reduce the data independently, following the procedure described in Puzia et al. (1999). In summary, the images were combined and cosmic ray cleaned within IRAF, and object magnitudes measured in 2 pixels apertures using SExtractor. Aperture correction derived in Puzia et al. (1999) for globular clusters in NGC 4472 (a Virgo galaxy at a similar distance to M87 and NGC 4478) were applied to correct for the extended nature of the clusters. The standard calibrations given in Holtzman et al. (1995) were used to compute Johnson $V$ and $I$ magnitudes. The results were compared with the lists of Whitmore et al. and Neilsen et al., and found to be in excellent agreement.

Globular cluster candidates were selected from the HST data using the image shape parameters returned by SEXTRACTOR. The optical and near-infrared lists were combined to final candidate lists, including 93 objects in M87 and 19 in NGC 4478. Their magnitudes are plotted against the total $V-K$ errors in Fig. 3. The limiting magnitudes for detection are roughly $K \sim 21$ and $K \sim 22$ for M87 and NGC 4478, respectively.
For some later applications, we will restrict the sample to clusters with errors in $(V-K)<0.16$ mag (see above), leaving 70 clusters in M87 and 14 in NGC 4478, and reducing the limiting magnitudes by $\sim 1$ mag in each case.

\subsection{Spectroscopy}

For M87, we checked the literature to see if any of the clusters for which we obtained a $K$-band magnitude had been included in a spectroscopic sample. Unfortunately, this was not the case, mainly due to the proximity of our fields to the centre of M87. On the other hand, this underscores an advantage of NIR studies that can probe, with high metallicity sensitivity, globular clusters in the inner regions of galaxies, inaccessible to spectroscopic studies.

\section{The $V, I, K$ colours of the globular clusters}

The colour-magnitude diagrams ( $V$ against $V-K$ ) for the globular cluster in M87 and NGC 4478 are shown in Fig. 4. We over-plotted models from Maraston (2000) as metallicity (age) references, and a line showing our equal detection probability: the data are complete down to $V>24$, however only to $K \leq 21$ and $K \leq 22$ for M87 and NGC 4478, respectively. Thus, we detect red clusters more easily than blue ones.

Throughout the paper we will compare the data with population synthesis models. We use models from Maraston (2000) and Bruzual \& Charlot (2000) to illustrate the differences between current models in the NIR. For detailed descriptions we refer the reader to the original papers, listing only the main differences briefly below. Both models use the same model atmospheres ("Basel atmospheres", Lejeune et al. 1998), but differ in the stellar evolution tracks: while Maraston uses tracks from the Frascati database (Cassisi et al. 1999 and references therein), Bruzual \& Charlot use the Padova tracks (Alongi et al. 1993; Girardi et al. 1996 and references therein). Both sets of tracks are overall very similar but differ in the slope of the AGB. Further, the main difference between the models is the fact that Maraston computes her models using the fuel consumption method (Renzini \& Buzzoni 1986) while Bruzual \& Charlot use the more classical isochrone synthesis method. The two last points taken together slightly affect the $K$ and $V-K$ predictions at high metallicities, as well as significantly affecting the isochrones for young ages $(<1 \mathrm{Gyr}$, see Maraston et al. 2000). The latter is, however, of no concern for this paper. The difference between the models is well illustrated in Fig. 10, where for $V-K>3$ the 15 Gyr Bruzual \& Charlot isochrone overlaps with the $9 \mathrm{Gyr}$ isochrone of Maraston. However, for ages younger than $\sim 9$ Gyr and high metallicities, both models predict the same trend. Thus the model uncertainties prevent us from reliably assigning absolute ages, but the choice of a particular model will not significantly affect our overall results.

The colour histograms are shown in Fig. 5. As indicated in Fig. 4, our observations are biased in favour of red clusters. Quantitatively, the following corrections should be applied. The peak magnitude of the Gaussian globular cluster luminosity function lies around $V_{\mathrm{TO}} \sim 23.8$ at the Virgo distance (e.g. Puzia et al. 1999), and is similar to within a few tenths of a 


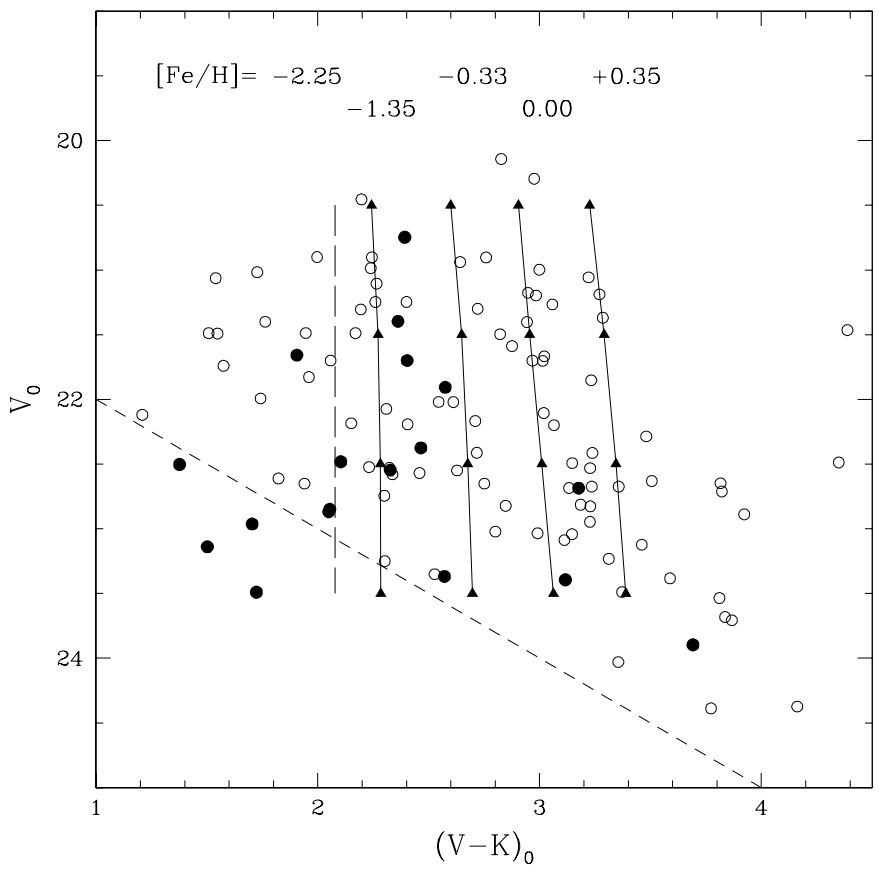

Fig. 4. Colour magnitude diagrams for globular clusters in M87 (open circles), and NGC 4478 (solid dots). The short dashed line indicates a line of equal detection probability (arbitrarily shifted here to $K=21$ which roughly corresponds the $(V-K)$ error limited adopted throughout the paper). For five metallicities, we show the predicted colours from simple stellar population models (here using Maraston 2000) for four ages $(15,13,11$, and $9 \mathrm{Gyr}$, from top to bottom, except for $[\mathrm{Fe} / \mathrm{H}]=-2.25$ for which only models for $15 \mathrm{Gyr}$ were available). The models points are arbitrarily shifted in magnitude (driven primarily by the mass and not the age of the clusters).

magnitude for blue and red clusters. Thus, we expect the peaks in $K$ to lie around $K_{\mathrm{TO}} \sim 21.6$ for blue $(V-K \sim 2.2)$ clusters, and $K_{\mathrm{TO}} \sim 20.7$ for red $(V-K \sim 3.2)$ clusters. The $V$ band luminosity function has a width of $\sigma=1.0$ to $1.2 \mathrm{mag}$, and is expected to be similar in $K$ for clusters in a narrow colour range.

For M87, our limiting $K$ magnitude lies around $21 \mathrm{mag}$, so that for clusters with $V-K \sim 2.2$ we fall short of the peak of the luminosity function by $\sim 0.5 \sigma$, i.e. sampling only around $30 \%$ of the blue population in the area covered. For red clusters, however, our data are deep enough to pass the peak of the luminosity function by $\sim 0.3 \sigma$, i.e. we sample around $60 \%$ of the red population in the area covered. Thus, in the case of M87, the blue side of the histogram should be corrected by roughly a factor of 2 when compared to the red side.

The effect is similar for NGC 4478. However, since our limiting magnitude lies around $K \sim 22$, in the area covered, we sample around $60 \%$ and $85 \%$ of the blue and red population, respectively. Thus the correction to apply when comparing blue and red numbers is smaller.

Finally, we show in Fig. 6 the $V-I$ against $V-K$ colours for the clusters, including the errors. Note that despite the fact that the $V-K$ photometric errors are larger than the $V-I$ ones, the ratio of the errors with respect to the colour range spanned is more favourable for $V-K$ by a factor of about 2 . Indeed, the mean error in $V-K$ is 0.098 mag for this sample $(0.129$

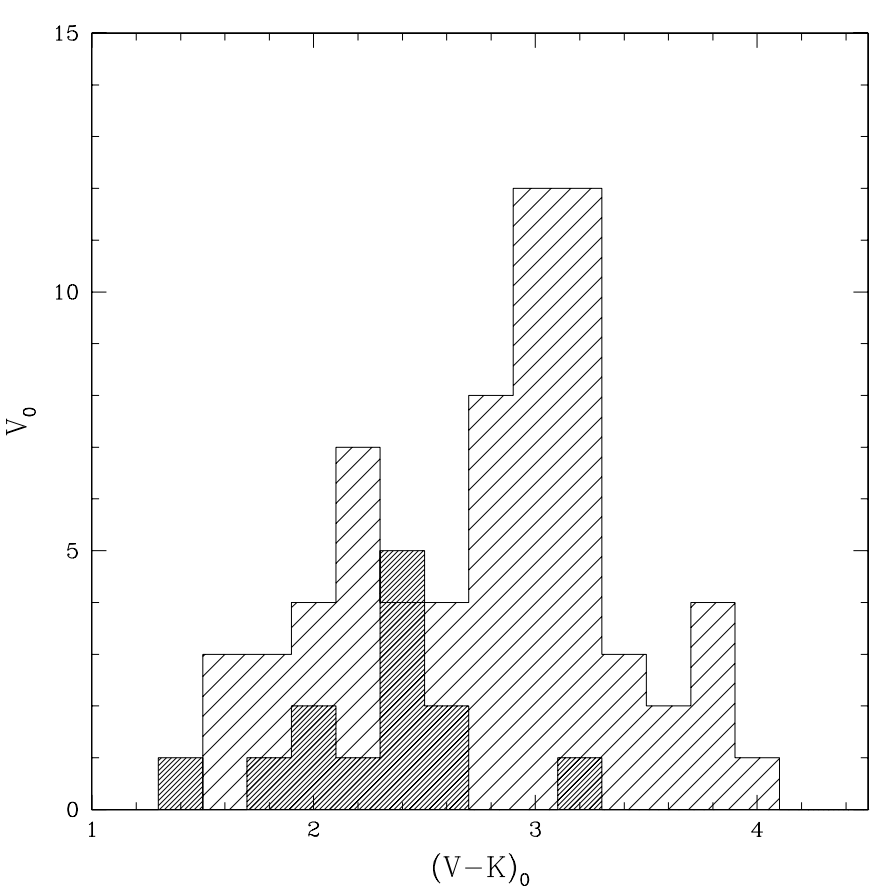

Fig. 5. $V-K$ histogram for M87 (light hashed) and NGC 4478 (dark hashed) globular clusters. Note that the histograms are biased in favour of red clusters (see text).

when the sample is not restricted to clusters with errors $<0.16$ ) for a range in colour of $>1.5 \mathrm{mag}$. The mean error in $V-I$ is $0.061 \mathrm{mag}$ (0.066 mag for the unrestricted sample) and the range in colour is $\sim 0.5$. Thus, the colour "resolution" in $V-K$ is $1.5 / 0.098 \simeq 15$, while in $V-I$ it is $0.5 / 0.061 \simeq 8$.

We will show further versions of Fig. 6 when comparing our data to Milky Way and M31 clusters, as well as to population synthesis models in Sect. 4.1.

\subsection{Transforming $(\mathrm{V}-\mathrm{K})$ into $[\mathrm{Fe} / \mathrm{H}]$}

An early empirical relation between $V-K$ and $[\mathrm{Fe} / \mathrm{H}]$ was proposed by Brodie \& Huchra (1990) based on Milky Way and M31 clusters, with the form: $[\mathrm{Fe} / \mathrm{H}]=1.574 \cdot(V-K)-5.110$, the scatter around this relation being on the order of $0.27 \mathrm{dex}$. Clusters with metallicities from $[\mathrm{Fe} / \mathrm{H}] \simeq-2.3$ dex to $[\mathrm{Fe} / \mathrm{H}] \simeq$ $-0.5 \operatorname{dex}(V-K<3.0)$ were available to define the relation.

A more recent calibration was proposed by Barmby et al. (2000), based on a newer compilation of Galactic clusters. They obtained shallower slopes for the relation (1.30, if only Galactic clusters with low reddening were used, 1.40 otherwise). Using their sample of M31 clusters they get a steeper slope with a relation of the form $[\mathrm{Fe} / \mathrm{H}] \propto \sim 1.90 \cdot(V-K)$.

Here we use all available Galactic and M31 clusters from the above compilations to derive a new relation. We consider only clusters with low reddening $(\mathrm{E}(B-V)<0.27$, including three quarters of the M31 data, and 129 data points in total). We obtain a linear relation of the form:

$[\mathrm{Fe} / \mathrm{H}]=1.82( \pm 0.11) \cdot(V-K)-5.52( \pm 0.26)$

(with an rms of 0.29 dex).

The data and relation are shown in Fig. 7. We also attempted to fit a second-order polynomial to the data and 

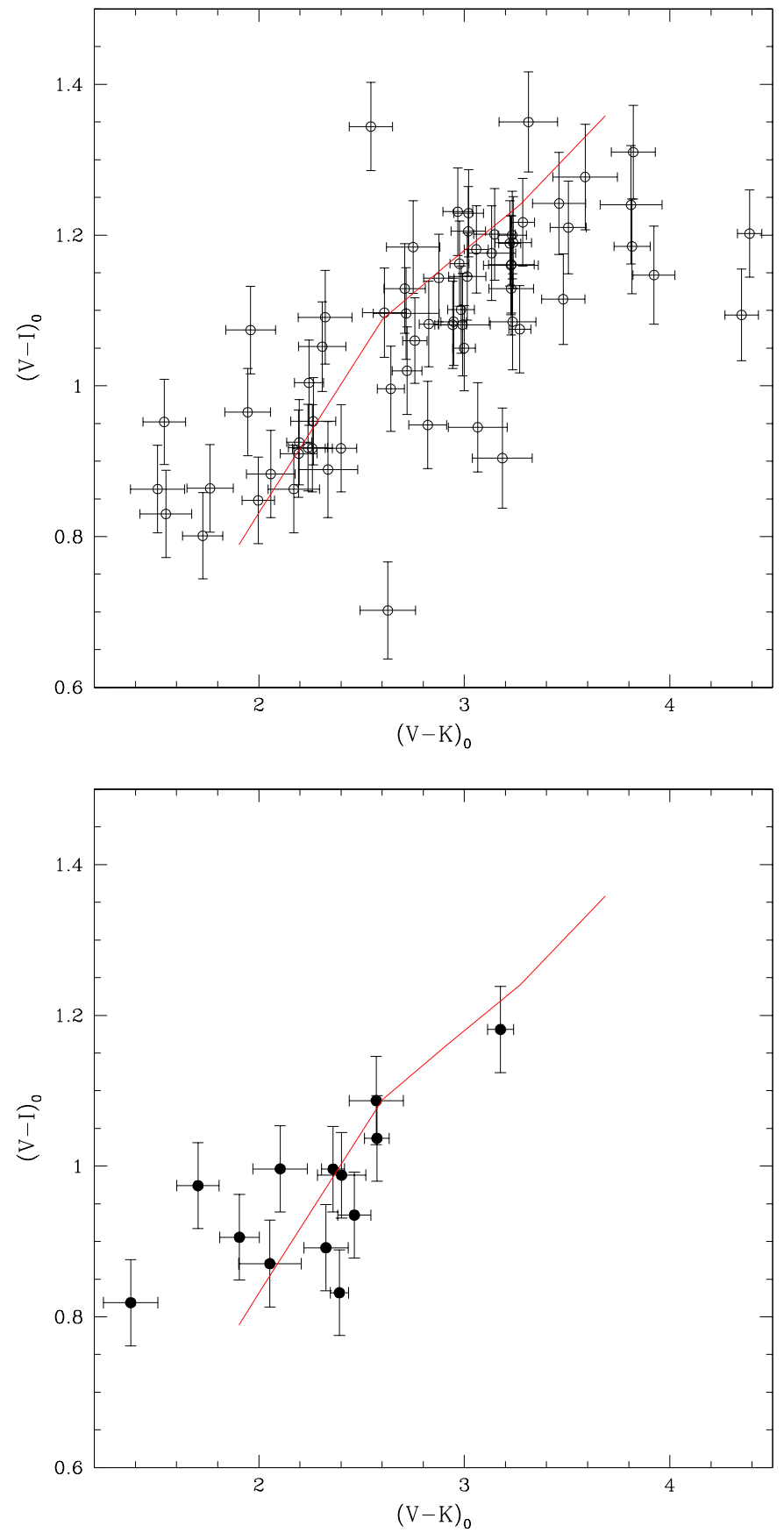

Fig. 6. Colour-colour diagram for globular clusters in M87 (upper panel) and NGC 4478 (lower panel). Only clusters with errors in $V-K$ less than $0.16 \mathrm{mag}$ are shown (all have errors in $V-I<0.1$ ). For comparison, a solid line shows a $15 \mathrm{Gyr}$ isochrone (from $1 / 200$ to twice solar metallicity) of a simple stellar population (using here Bruzual \& Charlot 2000 models).

obtained a best fit of the form: $[\mathrm{Fe} / \mathrm{H}]=-2.33( \pm 1.81)-$ $0.92( \pm 1.55) \cdot(V-K)+0.59( \pm 0.33) \cdot(V-K)^{2}$, with a standard deviation of the residuals of 0.29 , a reduced $\chi^{2}$ of 0.0855 and an $f$-test result of 134 , indicating a poor fit.

In the following, we will use our new linear relation, stressing that it is only valid in the colour range $1.8<(V-K)<3.0$ and metallicity range $-2.3<[\mathrm{Fe} / \mathrm{H}]<-0.2 \mathrm{dex}$, and that it requires uncertain extrapolations outside these ranges, especially

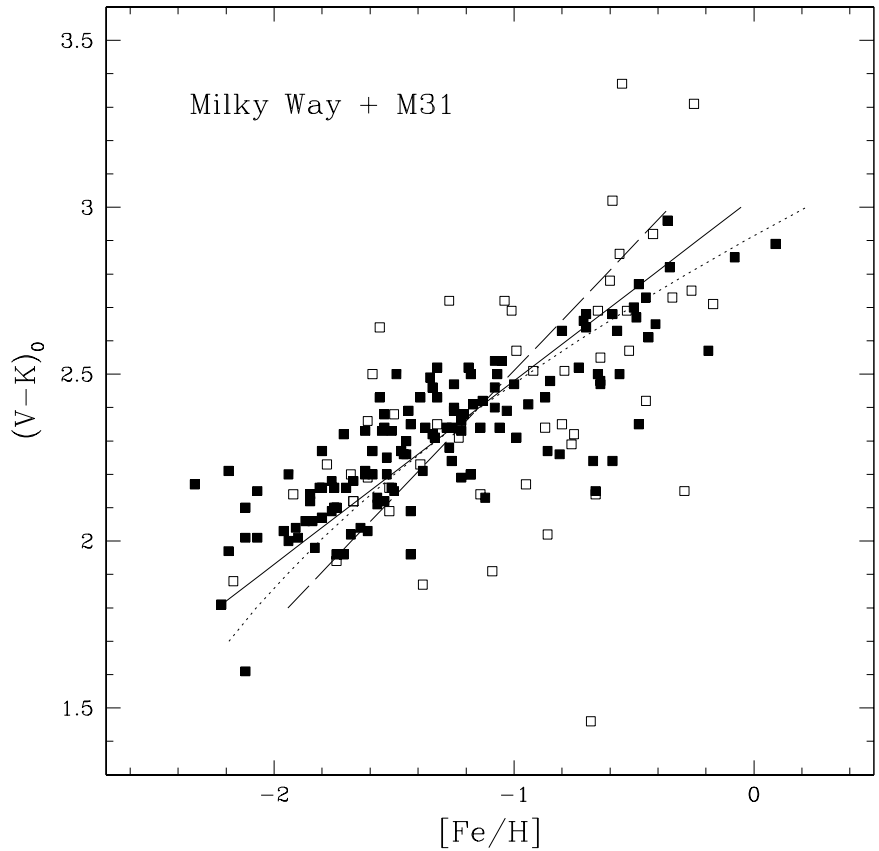

Fig. 7. Relation between $(V-K)$ and $[\mathrm{Fe} / \mathrm{H}]$ for globular clusters in the Milky Way and M31. Solid points indicate clusters with a reddening $E(B-V)<0.27$, open points clusters with higher reddening. Linear least squares fits to the low-reddening and total sample are indicated as solid and short-dashed lines, respectively. A second-order polynomial fit is shown as dotted line.

towards higher metallicities. There is a hint that the relation has a shallower slope at the high metallicity end. We will return to this point in Sect. 4.

\section{2. $\ln M 87$}

The colour distribution in M87 is broad, spanning over 2 mag in colour. Most globular cluster candidates span the range $1.8<$ $V-K<3.4$ with several candidates having colours as extreme as $V-K \sim 1.5$ and $V-K \sim 4.3$. As a comparison, the most metal-rich globular clusters in the Milky Way and M31 have colours of $V-K<3.2$.

\subsubsection{Very red globular cluster candidates}

Figure 8 shows the location within our NIR fields of the clusters with very red colours $(3.4<V-K<4.0)$. These objects were all individually inspected by eye on the WFPC2 images, but none showed any peculiarity that would disqualify it as a good globular cluster candidate. They do not seem to be spatially clumped nor are they concentrated towards the center of M87 (as verified by monte carlo simulations). Thus their morphological properties do not permit any useful conclusions to be drawn concerning the nature of these objects. Note that this group of clusters does not stand out in $V-I$ since their optical colours are not exceptionally red, and they blend in with the "normal" red tail of objects in the $V-I$ distribution. It is their $V-K$ colour that characterizes them. A final answer on their nature can only come from follow-up spectroscopy, 


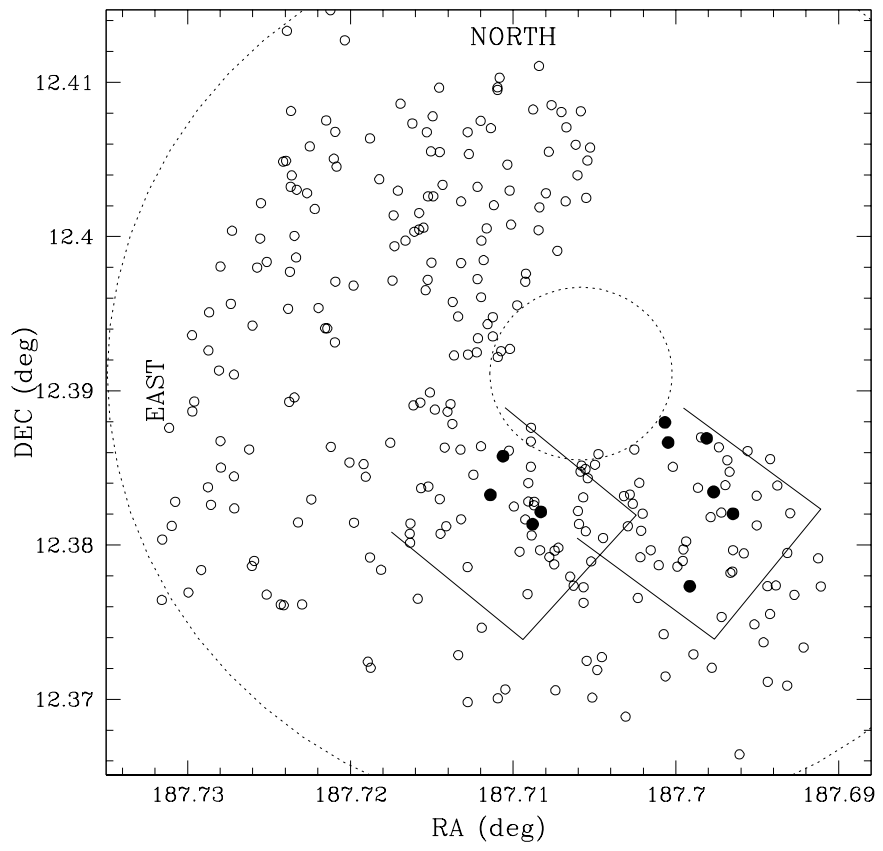

Fig. 8. Position of objects with $V-K>3.4$ in M87 (solid dots) within our $K$-band fields (3-sided frames) compared to the position of all objects detect on the WFPC2 images with colours $0.8<V-I<1.5$ and $21<V<23$ (open dots). The outer circle marks $1 R_{\text {eff }}\left(104^{\prime \prime}\right)$, the inner one $20^{\prime \prime}$.

for which purpose we give in Table 2 their magnitudes, colours and positions (as returned by the task metric within IRAF, i.e. relative positions probably good to $0.1^{\prime \prime}$ ).

\subsubsection{From $(\mathrm{V}-\mathrm{K})$ to $[\mathrm{Fe} / \mathrm{H}]$}

The colours can be translated into metallicity either using population synthesis models, or using empirical calibrations derived e.g. from the Milky Way or M31 globular clusters (see last section).

Using the empirical relation derived above, the globular clusters around M87 would have metallicities ranging from $[\mathrm{Fe} / \mathrm{H}]=-2.7$ dex to 0.75 dex. This broad range is supported by a comparison with population synthesis models (see Fig. 4).

The histogram of colours for clusters in M87 clearly shows two major peaks. This can be quantified by applying the KMM test (Ashman et al. 1993), which tests the hypothesis that a single Gaussian is a better fit than the sum of two Gaussians. The KMM test, run on our dataset selected for clusters with $V-K<4.0$ (87 objects), returns a probably of $P=0.066$, clearly favouring two Gaussians. The two colour peaks are found at $V-K=2.11 \pm 0.02$ and $V-K=3.13 \pm 0.02$. These colour peaks translate into metallicity peaks of $[\mathrm{Fe} / \mathrm{H}]=$ -1.68 dex using our relation derived in Sect. 3.1 ( -1.79 dex when using the Brodie \& Huchra relation) for the metal-poor clusters, and of $[\mathrm{Fe} / \mathrm{H}]=+0.18 \mathrm{dex}(-0.18 \mathrm{dex})$ for the metalrich clusters (all values with total errors around $0.3 \mathrm{dex}$ ).

M87 has long been known to host (at least) two distinct populations (e.g. Lee \& Geisler 1993; Whitmore et al. 1995). The most recent study by Larsen et al. (2000) found the two peaks in $V-I$ at 0.95 and 1.20 mag (within errors of a few hundreds of a magnitude), which translate into metallicity peaks of $[\mathrm{Fe} / \mathrm{H}] \sim-1.4$ and $-0.6 \mathrm{dex}$, when using the relation given by Kissler-Patig et al. (1998), or $[\mathrm{Fe} / \mathrm{H}] \sim-1.3$ and 0.0 dex, when adopting the relation from Couture et al. (1990). Thus, the combination of NIR and optical colours suggest a slightly more metal-poor blue sub-population and a slightly more metal-rich red sub-population in M87.

\subsection{In NGC 4478}

The colour distribution of globular clusters in NGC 4478 is very different from the one in its giant neighbour. About 18 globular cluster candidates were detected in our field, compared to a total population of $\sim 40$, derived by Neilsen et al. (1997). As in the optical study, hardly any red globular clusters were detected, despite the fact that our observations were more sensitive to red objects.

A KMM test for bi-modality on the small sample is inconclusive. The mean $V-K$ colour of the sample is $2.31 \pm$ $0.03 \mathrm{mag}$ (and a dispersion $\sigma=0.60 \mathrm{mag}$ ). This corresponds to a metallicity of $[\mathrm{Fe} / \mathrm{H}]=-1.32$ dex using our relation of Sect. 3.1 (-1.47 dex when using the Brodie \& Huchra relation). Computing the mean for the sample bluer than $V-K=3.0$ (15 objects, leaving out the 1 red cluster) returns a mean colour of $V-K=2.10 \pm 0.03$, with dispersion of 0.39 mag. This corresponds to a metallicity of $[\mathrm{Fe} / \mathrm{H}]=-1.70 \mathrm{dex}(-1.80 \mathrm{dex})$. Identical to the blue sub-population in M87.

\section{Discussion}

\subsection{A comparison between M87, NGC 4478, and the Local Group systems}

In the following, we compare the colour/metallicity distributions of M87 and NGC 4478 to the ones of the Milky Way and M31. Figure 9 shows a colour-colour plot for all four samples. Note that the Local Group samples are biased towards metalpoor clusters, with the most metal-rich ones having metallicities around $[\mathrm{Fe} / \mathrm{H}] \sim-0.3 \mathrm{dex}$.

For M87 the combination of optical and near-infrared colours showed more clearly than the optical colours alone, that the giant galaxy hosts extreme clusters when compared to the Milky Way and M31. While all the clusters in the colour-colour diagram lie on the relationship defined by the Local Group galaxies, M87 extends this relation to extremes. The blue globular clusters extend to slightly bluer $V-K$ colours than the bulk of halo cluster in the Local Group, while the red clusters are on average redder in $V-K$ than even the reddest Local Group globular clusters.

The study of a large sample of galaxies showed that the populations of metal-poor clusters appears to have a fairly universal average metallicity around $[\mathrm{Fe} / \mathrm{H}] \sim-1.4 \pm 0.2$ (mostly derived from optical colours alone: Burgarella et al. 2001; Larsen et al. 2001) with very little dispersion from galaxy to galaxy. The wider baseline of the $V-K$ colour suggests that M87 has, on average, very metal-poor clusters in its metalpoor population (if colour is interpreted solely in terms of metallicity). 
Table 2. Very red clusters in M87.

\begin{tabular}{lrcccc}
\hline \hline ID & $\Delta \mathrm{RA}$ & $\Delta \mathrm{Dec}$ & $V_{0}[\mathrm{mag}]$ & $(V-I)_{0}[\mathrm{mag}]$ & $(V-K)_{0}[\mathrm{mag}]$ \\
\hline KPBMred1 & 16.8 & -19.3 & $22.704 \pm 0.043$ & $1.094 \pm 0.061$ & $4.35 \pm 0.08$ \\
KPBMred2 & 19.6 & -28.3 & $23.600 \pm 0.051$ & $1.277 \pm 0.070$ & $3.59 \pm 0.16$ \\
KPBMred3 & 10.5 & -35.1 & $21.681 \pm 0.041$ & $1.202 \pm 0.058$ & $4.39 \pm 0.06$ \\
KPBMred4 & 8.7 & -32.3 & $23.105 \pm 0.046$ & $1.147 \pm 0.065$ & $3.92 \pm 0.10$ \\
KPBMred5 & -32.8 & -11.4 & $22.865 \pm 0.045$ & $1.185 \pm 0.063$ & $3.82 \pm 0.09$ \\
KPBMred6 & -28.7 & -32.7 & $23.340 \pm 0.049$ & $1.242 \pm 0.068$ & $3.46 \pm 0.13$ \\
KPBMred7 & -27.1 & -27.6 & $22.503 \pm 0.043$ & $1.115 \pm 0.060$ & $3.48 \pm 0.11$ \\
KPBMred8 & -18.8 & -15.1 & $23.752 \pm 0.057$ & $1.240 \pm 0.079$ & $3.81 \pm 0.15$ \\
KPBMred9 & -13.3 & -16.1 & $22.848 \pm 0.044$ & $1.210 \pm 0.062$ & $3.51 \pm 0.09$ \\
KPBMred10 & -23.5 & -49.6 & $22.929 \pm 0.045$ & $1.310 \pm 0.062$ & $3.82 \pm 0.11$ \\
\hline
\end{tabular}

The positions are given relative to the centre of M87: $12 \mathrm{~h} 30 \mathrm{~m} 49.4 \mathrm{~s}+12 \mathrm{~d} 23 \mathrm{~m} 28 \mathrm{~s}$ (J2000).

The metal-rich clusters in our M87 sample are found to have around solar metallicities (see also Fig. 4). $V-I$ studies already suggested this result, but are affected by transformation uncertainties between colour and metallicity. In addition, the colour-metallicity relation in the optical flattens at high metallicities which makes it even more difficult to detect very metalrich clusters in $V-I$. The $V-K$ colour allows a direct comparison with Milky Way and M31 globular clusters and clearly shows the redder colours of the M87 clusters. This trend is stronger in $V-K$ than in $V-I$ which appears to "saturate" at high metallicities.

NGC 4478 hosts an almost exclusively blue population of globular clusters (see above). However, when compared to the Milky Way and M31, the population is very similar in $V-I$ and $V-K$ to the (halo) populations of the Local Group galaxies. Also, the mean metallicity is in very good agreement with the "universal" value found for metal-poor populations in other early-type galaxies.

\subsection{A comparison with SSP models}

The sample of globular clusters around M87 is well-suited to testing population synthesis models in the near infrared. As seen in Fig. 9, the Local Group simple stellar populations (i.e. globular clusters) do not provide samples extending to very red $(V-K>3.0)$ colours.

Overall, the clusters are well-traced in the colour-colour plot by a $15 \mathrm{Gyr}$ isochrone. However, for colours redder than $V-K>3.0$, the clusters seem to deviate from the old models. The red clusters lie "below" the old isochrone (e.g. see Fig. 6).

In Fig. 10, we show a set of younger isochrones (9 to $15 \mathrm{Gyr}$ ), but these also fail to match the reddest data points. Only when extending the isochrones to very high metallicities (twice solar) and allowing for younger ages (from 1 to 8 Gyr), the models occupy the region populated by the reddest M87 clusters. According to the models, these clusters would have solar to twice solar metallicities and ages between 2 and $8 \mathrm{Gyr}$.

At these younger ages, a cluster would be expected to be 0.5 to 1 mag brighter than an old (15 Gyr) cluster of the same mass and metallicity. This luminosity difference is too small to allow us to use the apparent magnitudes as age discriminators. We only notice that the reddest clusters are among the faintest detected clusters, although still around the visual turn-over magnitude of the globular cluster luminosity function. If the reddest clusters have a mass distribution similar to the blue and red ones, we can exclude the existence of a large number of them since we would have detected many more at brighter magnitudes.

To quantify the fraction of intermediate-age clusters is difficult. A simple attempt can be made by comparing the number counts of clusters compatible with being old and metal-rich with the number of intermediate-age candidates. The association of a cluster with the one or the other group is uncertain, and we try to bracket the ratio with two extreme cases. For both cases, we base our counts on the data as shown in Fig. 10, with $(V-K)_{0}>2.6$ in order to exclude the old, metal-poor subpopulation. In the first case, we consider only clusters on the twice solar track with ages $<5$ Gyr to be of intermediate age. In this case, we obtain an old/young ratio of $4 / 1$. In the second case, we consider only clusters on $>9 \mathrm{Gyr}$ isochrones and up to solar metallicity to be old and metal-rich. In that case, we obtain a ratio old/young of $1 / 1$. These ratios must be corrected for two biases: $i$ ) the intermediate age clusters are on average redder and thus less affected by incompleteness; and ii) the intermediate-age clusters will be up to 1 mag brighter, i.e. we sample a larger fraction of the total sub-population (assuming that old and intermediate age clusters have the same mass function). These two effects amount to $10-100 \%$ correction, mostly depending on the exact age and mean colour of the intermediate age clusters. The fraction of intermediate-age clusters in our sample is thus estimated to be between $10 \%$ and $50 \%$. Finally, these number counts are made on a small area close to the center of the galaxy. Therefore statistical fluctuations as well as different spatial distributions can further change the ratios for the overall globular cluster system of M87.

Would clusters with such young ages have been detected in the spectroscopic studies? Indeed, Brodie \& Huchra (1991), Kissler-Patig et al. (1998), Cohen et al. (1998) and Forbes et al. (2002) have reported a few clusters in early-type galaxies with unusually high Balmer-line strengths for their metallicity, possibly indicating young ages. These clusters were among the reddest of the sample, although optical colours alone do 
not allow their identification as extremely red. Also, the agemetallicity degeneracy, as well as uncertainties in the structure of the horizontal branches prevent definitive disentangling of age and metallicity effects using spectroscopic line indices alone.

In summary, the metal-poor globular clusters are very well represented by the models, which are very similar in the $(V-I)-(V-K)$ plane for a wide age range $(8-15 \mathrm{Gyr})$ at low metallicities. The bulk of the metal-rich clusters $(V-K<3.0)$ are also well represented by the models and are compatible with old isochrones. Any systematic age difference with respect to the metal-poor globular clusters is very model dependent and is in any case smaller than a few Gyr. For the reddest $(V-K>3.0)$ globular clusters in M87, old isochrones can no longer fit the data, and the models seem to indicate the presence of very metal-rich (>solar), intermediate age (2-8 Gyr) clusters. The colours for these clusters are comparable to opticalNIR colours of the confirmed intermediate age (few Gyr) clusters in NGC 1316 (Goudfrooij et al. 2001). The very reddest $(V-K \sim 4)$ clusters remain a puzzle in terms of simple stellar population models, but resemble the objects in Cen A suspected to be young-to-intermediate age star clusters (Minniti et al. 1996).

\subsection{Where are NGC 4478's red clusters?}

NGC 4478 appears to have a normal metal-poor globular cluster sub-population but its lack of metal-rich clusters is surprising. Other early-type galaxies are known to be dominated by metal-poor globular clusters, but they also host a significant

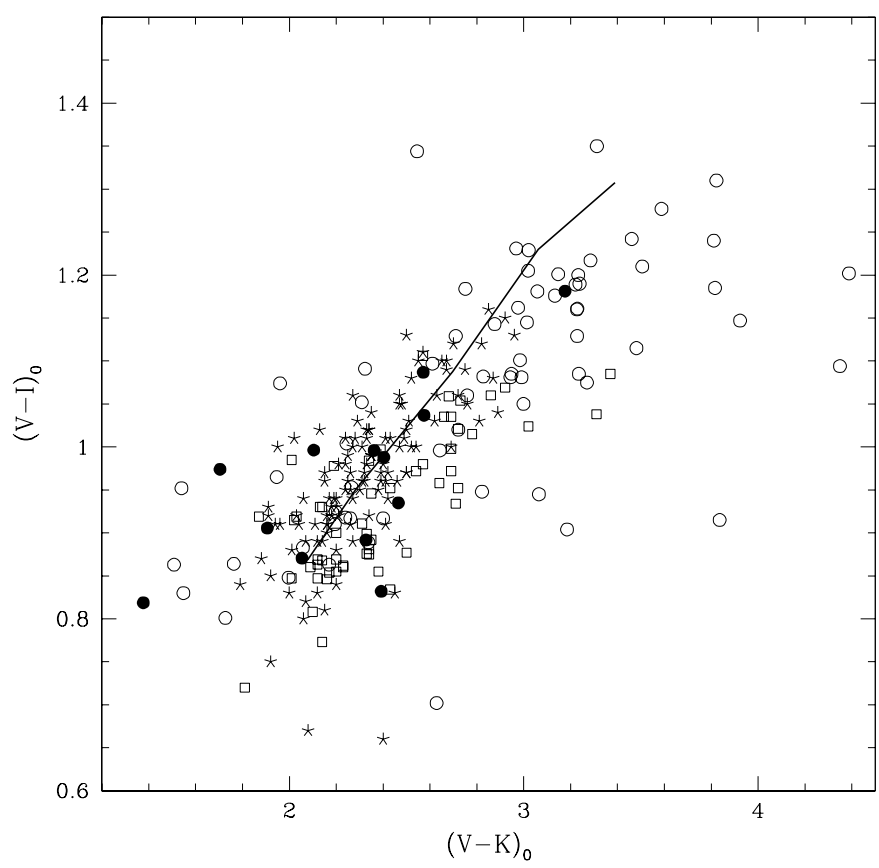

Fig. 9. Colour-colour diagram showing globular clusters of M87 (open dots), NGC 4478 (solid dots), the Milky Way (open squares) and M31 (stars). For comparison, a solid line shows a $15 \mathrm{Gyr}$ isochrone (from $1 / 200$ to twice solar metallicity) of a simple stellar population (using here Maraston (2000) models). population of metal-rich clusters. The origin of such metalpoor dominated distributions is still widely debated but is usually attributed to the "mix" of formation scenarios, namely accretion, disk mergers, or in situ formation (see Gebhardt \& Kissler-Patig 1999; Brodie et al. 2000). To date, only one other giant elliptical has been shown to host exclusively metal-poor globular clusters: NGC 4874, the central cD galaxies in Coma (see Harris et al. 2000). We will return to this case at the end of this section.

The immediate question is whether NGC 4478 also qualifies as galaxy with exclusively metal-poor globular clusters. That is, does NGC 4478 host any red globular clusters at all? Our study shows 1 globular cluster redder than $V-K=2.6$ (which is roughly the limit for "halo" clusters in the Milky Way and M31). The expected contamination from M87 at this galactocentric distance, according to the work of McLaughlin, Harris \& Hanes (1994) is around 6 clusters per $\operatorname{arcmin}^{2}$ down to $V=24$ mag (roughly our detection limit in the red). The ratio of blue to red clusters at this large galactocentric distance is around 2/1 (estimated for NGC 4472 by Rhode \& Zepf 2001). Therefore, we expect 1 to 2 red clusters belonging to M87 over our total area of $\sim 0.8 \operatorname{arcmin}^{2}$ around NGC 4478. Thus, the one red globular cluster seen around NGC 4478 is fully compatible with contamination from M87. Similar arguments can be made from the optical data alone (e.g. Neilsen et al. 1997). NGC 4478 is likely to have no red globular clusters at all.

NGC 4478 is a "normal" elliptical galaxy with respect to known scaling laws ( $\mathrm{Mg}-\sigma$ relation, fundamental plane), and ranks among the dozen brightest galaxies of the Virgo

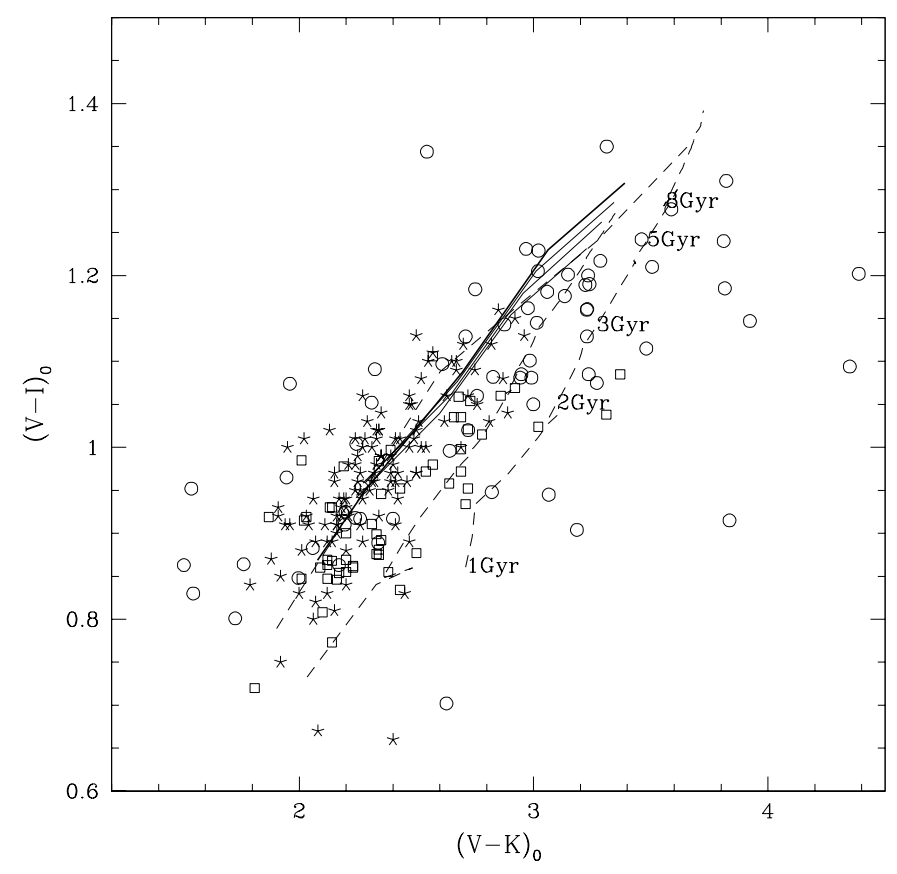

Fig. 10. Colours of M87 globular clusters compared with models. The solid lines show isochrones (from 1/200th to twice solar) of 15,13 , 11 and 9 Gyr (models from Maraston 2000). The dashed lines show a $15 \mathrm{Gyr}$ isochrone as well as two iso-metallicity lines (solar and twice solar from 1 to $15 \mathrm{Gyr}$ ) from Bruzual \& Charlot (2000). Other symbols as in Fig. 9. 
cluster. It stands out only as being a likely companion of M87. The projected distance between the two galaxies is $8.5^{\prime}$, i.e. $\sim 40 \mathrm{kpc}$ (assuming a distance to both galaxies of $16 \mathrm{Mpc}$ ), well inside the extended $\mathrm{cD}$ halo of M87 in projection (e.g. Carter \& Dixon 1978). NGC 4478 has been classified as a compact elliptical (Prugniel et al. 1987) because of its lack of an extended envelope, and the influence of M87 is evident from the large twist of its outer isophotes (Peletier et al. 1990; Michard 1985; Caon et al. 1990), and the complex nuclear morphology (van den Bosch et al. 1994). Thus, NGC 4478 is likely to have been tidally truncated by M87. Could this be the explanation for the absence of red clusters?

A priori, the red clusters are more concentrated than the blue ones, so that truncation would be expected to affect, if anything, the blue population preferentially. The specific frequency of NGC 4478 is very low $\left(S_{N} \sim 0.6\right.$, Neilsen et al. 1997), which would be a consequence of the outer parts (rich in clusters but poor in stars) having been stripped. However, the complete absence of red clusters leads us to speculate that the tidal truncation was effective before the formation of the red clusters. In a hierarchical formation scenario the first small structures (responsible for the formation of blue clusters) were able to form and assemble, but the remaining gas from this phase could have been stripped before or during the formation of the red population and associated stars, hindering their efficient formation. Such a scenario implies a low fraction of old metal-rich stars with respect to old metal-poor stars. This is supported by the somewhat low metallicity and high $\mathrm{H}_{\beta}$ of the galaxy's integrated light (Gonzales 1993) which can be interpreted as the presence of a high fraction of old metal-poor stars (Maraston \& Thomas 2000), matching the globular cluster population. Interestingly, NGC 4478 has a low $\alpha$-element enrichment as judged from the $\mathrm{Mg}_{2} /<\mathrm{Fe}>$ ratio, which hints at star formation on long timescales rather than a rapid collapse. This could in turn explain the high fraction of (metal-rich) stars (i.e. low $S_{N}$ ). NGC 4478 appears to have built up a metal-rich stellar population without forming metal-rich globular clusters. This may indicate a peculiar star-formation history/mechanism compared to other gE galaxies. A systematic study of the stellar and globular cluster populations of tidally truncated galaxies would be of great interest for understanding the early stages of galaxy formation/assembly.

Getting back to the case of NGC 4874: the galaxy differs from NGC 4478 in the sense that instead of being influenced by a central galaxy, the former dominates its environment, and tidal truncation by a neighbour seems unlikely. However, Harris et al. observe a similar contradiction (maybe even more severe in their case); the galaxy built up a significant metalrich stellar population without forming any metal-rich clusters. Harris et al. arrived at a similar conclusion; a mechanism must have acted to suppress the effective formation of massive globular clusters during the formation of the "bulge". They actually argue that a mechanism that would prevent clusters more massive than $10^{5} M_{\odot}$ from forming would also lead to the observed properties. In our case the optical data sample the globular cluster luminosity function almost completely, pushing down the limit to a few $10^{4} M_{\odot}$ (see also Neilsen \& Tsvetanov 1997). Any further comparison between the two galaxy remains, however, difficult given their very different natures and probably formation histories (central cD vs. truncated giant elliptical).

\subsection{The non-logarithmic metallicity distribution of $M 87$}

Finally, we ask whether the red sub-population in M87 itself shows sub-structure. In other words, whether the population of red clusters in M87 was built up by several mechanisms/events, or whether the bulk of the red globular clusters formed in a single event.

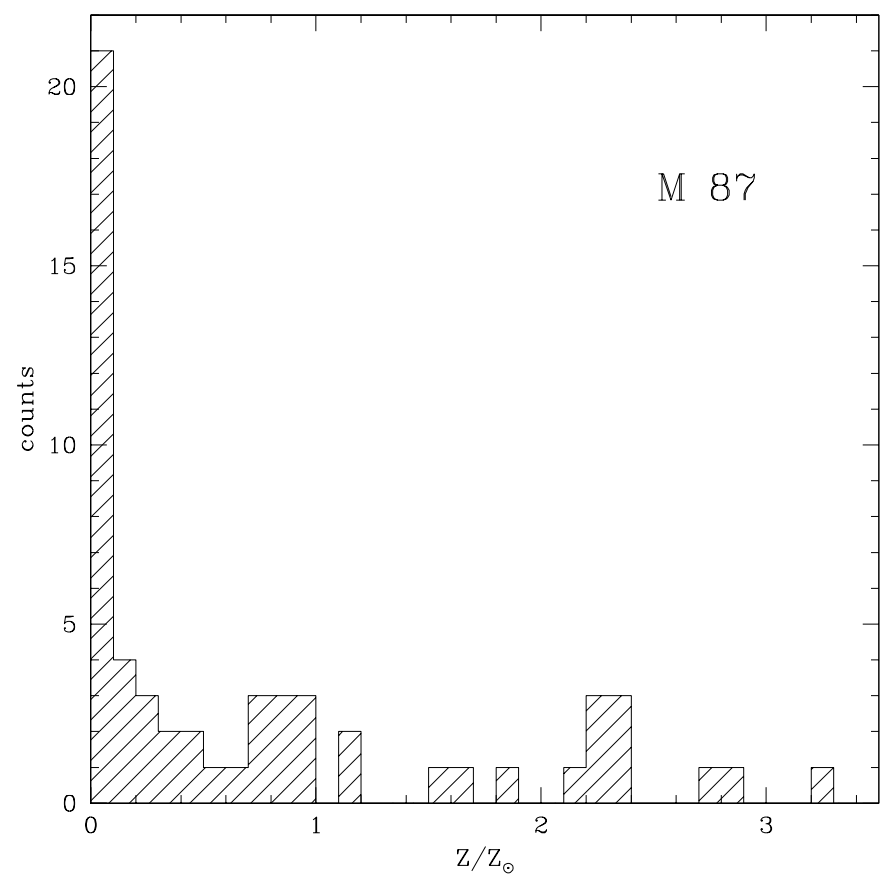

Fig. 11. Metalicity distributions $\left(Z / Z_{\odot}\right)$ of globular clusters in $M 87$. Note that beyond $Z / Z_{\odot}=1$ the conversion from colour to metallicity is based on an extrapolation of the empirical relations.

The colour histogram (see Fig. 5, equivalent to $[\mathrm{Fe} / \mathrm{H}]$ given the linear correspondence) shows the distribution of a logarithmic metallicity value. In Fig. 11 we show how different such metallicity distributions would look in linear metallicity $Z$. In the case of M87, the two clear peaks in $[\mathrm{Fe} / \mathrm{H}]$ (transformed linearly from $V-I$ ) disappear when plotted in $Z$. For this particular choice of zero point ( solar $=1$ ), the blue peak gets "compressed" into an even clearer peak between 0 and $0.1-0.2 Z_{\odot}$. The (typical) gap in colour at $[\mathrm{Fe} / \mathrm{H}] \sim-1$ dex can still be guessed at $Z \sim 0.1$, since it marks the clear cut from the peaked to the roughly flat distribution. The red peak gets spread over several tenths in $Z$ and is not recognizable as a single peak anymore.

We conducted simple simulations to determine to what extent the disappearance of the red peak was due to the fact that the (symmetric) photometric errors are asymmetrically distributed in the $Z$ plot. We simulated the M87 colour distribution using the peak location, peak width, total numbers, and blue to red number ratios returned by the KMM test. Each simulated data point was smeared by an error drawn randomly from 
our observed list of errors in $V-K$. The resulting 1000 artificial distributions were compared to the original data using a Kolmogorov-Smirnov test, in order to test whether a system composed by 2 sub-populations could reproduce the observed $Z$ distribution.

We varied the peak locations, the peak width, and the number ratio of blue to red clusters. We did this for a total of about 20000 simulated distributions. If the intrinsic widths of the sub-population was kept narrow (not significant when compared to the photometric errors, here $\sigma<0.2 \mathrm{mag}$ ) the resulting distribution completely failed to reproduce the observed one (the effect being more severe for the red ones). This excludes the possibility that the globular cluster system of M87 is built from two sub-populations of very narrow metallicity range. This is certainly true for the red sub-population, while this representation is not very sensitive to the intrinsic width of the blue sub-population in $[\mathrm{Fe} / \mathrm{H}]$. The best fit was obtained by allowing the red population to have a intrinsic width of 0.25 to $0.30 \mathrm{mag}$ in $V-K$ (i.e. $\sigma \sim 0.5 \mathrm{dex}$ in $[\mathrm{Fe} / \mathrm{H}]$ ), although even in that case the confidence level of the test was only around $80 \%$, on average. Unfortunately, our number statistics were not high enough to probe more complicated metallicity distributions, or 3 sub-populations. But we conclude from these data, that the red distribution has a spread in metallicity of $\sim 0.5 \operatorname{dex}(1 \sigma)$ roughly from half-solar to twice solar, or even wider if age affects the colours too (see Sect. 4.2). This spread in metallicity gets "played down" in the colour and $[\mathrm{Fe} / \mathrm{H}]$ distributions.

Thus, the interpretation of the metal-rich peak appears more complicated than originally assumed and the red population might well host multiple sub-populations. The large spread in $V-K$ is naturally explained by several successive events of red cluster formation, induced by successive gas-rich mergers as assumed in hierarchical formation scenarios. These dissipational events must have occurred at high redshifts (given that most clusters appear to be old), but a small fraction of them could have happened more recently $(z \sim 0.5-1)$ given the potential presence of intermediate age (few Gyr) clusters (see Sect. 4.2). The bulk of the star/cluster formation in M87 would nevertheless have occurred at high redshifts.

Alternatively, the large spread in $V-K$, i.e. in metallicity, is also compatible with a narrow age distribution, as expected if the metal-rich stellar and cluster populations had formed in a single event (in situ collapse or one single major merger). This would require extremely fast chemical enrichment during this major star/cluster formation event, so that almost coeval clusters could have very different metallicities. The presence of intermediate age clusters contradicts this hypothesis, i.e. the formation of the metal-rich component in a single collapse. The current data favour a more complex star/cluster formation history for M87 with several major star formation events having played a role from high to intermediate redshifts.

\section{Summary and conclusions}

We have compared optical and near infrared colours of globular clusters in M87, the central giant elliptical in Virgo, and NGC 4478, an intermediate luminosity galaxy in Virgo, close in projection to M87.
We find the broad range in colour and previously detected bi-modality in M87. We confirm that NGC 4478 only hosts a blue sub-population of globular clusters and now show that these clusters' $V-I$ and $V-K$ colours are very similar to those of the halo globular clusters in Milky Way and M31. Most likely, a metal-rich sub-population never formed around this galaxy (rather than having formed and been destroyed later), perhaps because its metal-rich gas was stripped during its passage through the centre of the Virgo cluster, or because it was subjected to some other mechanism that would prevent the formation of massive globular clusters during the formation of the "bulge".

In M87 the $V-I / V-K$ colours are close to those predicted from SSP models for old populations. However, M87 also hosts a few red clusters that are best explained by intermediate ages (few Gyr). Generally, there is evidence that the red, metal-rich sub-population has a complex structure and is itself composed of clusters spanning a large metallicity (from the spread in $V-K$ ) and, potentially, age range (from the spread in the $V-I-V-K$ diagram). This contrasts with the blue, metal-poor population which appears more homogeneous from galaxy to galaxy.

While the blue clusters still appear to be predominantly a single physical group, this may not be true for the red clusters. This prompts us to ask whether the metal-rich sub-group is, in many or most cases, just an amalgamation of multiple metalrich sub-populations that do not necessarily share the same origin. This would imply a complex star/cluster formation history, i.e. the origin of the metal-rich stellar population in early-type galaxies may not be just a single collapse or a single major merger but rather several such events.

Acknowledgements. We would like to thank Claudia Maraston and Stephane Charlot for providing their population synthesis models prior to publication.

MKP gratefully acknowledges the support of the Alexander von Humboldt Foundation through a Feodor Lynen Fellowship in the early stages of this project. DM is supported by Fondecyt, Fonfap, and Milenio, and he also thanks the ESO Visitor's program. Part of this research was funded by the National Science Foundation grant number AST-9900732 and HST grant GO.06554.01-95A.

The authors wish to extend special thanks to those of Hawaiian ancestry on whose sacred mountain we are privileged to be guests. Without their generous hospitality, none of the observations presented herein would have been possible.

\section{References}

Alongi, M., Bertelli, G., Bressan, A., Chiosi, C., \& Fagotto, F. 1993, A\&AS, 97, 851

Ashman, K. M., Bird, C. M., \& Zepf, S. E. 1994, AJ, 108, 2348

Ashman, K. M., \& Zepf, S. E. 1998, Globular Cluster Systems (Cambridge University Press)

Barmby, P., Huchra, J. P., Brodie, J. P., et al. 2000, AJ, 119, 727

Bertin, E., \& Arnouts, S. 1996, A\&AS, 117, 393

van den Bosch, F. C., Ferrarese, L., Jaffe, W., et al. 1994, AJ, 108, 1579

Brodie, J. P., \& Huchra, J. P. 1990, ApJ, 362, 503

Brodie, J. P., \& Huchra, J. P. 1991, ApJ, 379, 157

Brodie, J. P., Larsen, S. S., \& Kissler-Patig, M. 2000, ApJ, 543, L19 
Bruzual, A. G., \& Charlot, S. 2000, based on 1996, electronically available see: Leitherer, C., et al. 1996, PASP, 108, 996

Burgarella, D., Kissler-Patig, M., \& Buat, V. 2001, AJ, 121, 2647

Caon, N., Capaccioli, M., \& Rampazzo, R. 1990, A\&AS, 86, 429

Carter, D., \& Dixon, K. L. 1978, AJ, 83, 574

Cassisi, S., Castellani, V., degl'Innocenti, S., Salaris, M., \& Weiss, A. A\&AS, 134, 103

Cohen, J. G., Blakeslee, J. P., \& Ryzhov, A. 1998, ApJ, 496, 808

Couture, J., Harris, W. E., \& Allwright, J. W. B. 1990, ApJS, 73, 671

de Vaucouleurs, G., de Vaucouleurs, A., Corwin, H. G., et al. 1991, Third Ref. Catalogue of Bright Galaxies (Springer, New York)

Forbes, D. A., Beasly, M., Brodie, J. P., \& Kissler-Patig, M. 2002, ApJL in press

Frogel, J. A., Persson, S. E., \& Cohen, J. G. 1980, ApJ, 240, 785

Gebhardt, K., \& Kissler-Patig, M. 1999, AJ, 118, 1526

Girardi, L., Bressan, A., Chiosi, C., Bertelli, G., \& Nasi, E. 1996, A\&AS, 117, 113

Gonzales, J. J. 1993, Ph.D. Thesis, University of California in Santa Cruz

Goudfrooij, P., Alonso, M. V., Maraston, C., \& Minniti, D. 2001, MNRAS, 328, 237

Harris, W. E. 2001, Star Clusters, Saas-Fee Advanced Course 28 (Berlin, Heidelberg: Springer)

Harris, W. E., Kavelaars, J. J., Hanes, D. A., et al. 2000, ApJ, 533, 137

Holtzman, J., et al. 1995, PASP, 107, 1065

Kissler-Patig, M. 2000, Rev. Mod. Astron., Vol. 13, ed. Schielicke, p. 13

Kissler-Patig, M. 2002, Extragalactic Star Clusters, ed. D. Geisler, E. K. Grebe, \& D. Minniti, ASP Conf. Ser.

Kissler-Patig, M., Brodie, J. P., Schroder, L. L., et al. 1998, AJ, 115, 105

Kundu, A. 2000, Ph.D. Thesis, University of Maryland

Kundu, A., Whitmore, B. C., Sparks, W. B., et al. 1999, ApJ, 513, 733

Kundu, A., \& Whitmore, B. C. 2001a, AJ, 121, 2950
Kundu, A., \& Whitmore, B. C. 2001b, AJ, 122, 1251

Larsen, S. S., Brodie, J. P., Huchra, J. P., et al. 2001, AJ, 121, 2974

Lee, M. G., \& Geisler, D. 1993, AJ, 106, 493

Lejeune, T., Cuisinier, F., \& Buser, R. 1998, A\&AS, 130, 65

Maraston, C. 2000, priv.communication, based on Maraston, C. 1998, MNRAS 300, 872

Maraston, C., \& Thomas, D. 2000, ApJ, 541, 126

Maraston, C., Kissler-Patig, M., Brodie, J. P., et al. 2001, A\&A, 370, 176

Matthews, K., \& Soifer, B. T. 1994, Infrared Astronomy with Arrays: the Next Generation, ed. I. McLean (Dordrecht: Kluwer Academic Publishers), 239

McLaughlin, D., Harris, W. E., \& Hanes D. A. 1994, ApJ, 422, 486

Michard, M. 1985, A\&AS, 59, 205

Minniti, D., Alonso, M. V., Goudfrooij, P., et al. 1996, ApJ, 467, 221

Neilsen, E. H. Jr., Tsvetanov, Z. I., \& Ford, H. C. 1997, ApJ, 483, 745

Persson, S. E., Aaronson, M., Cohen, J. G., et al. 1983, ApJ, 266, 105

Persson, S. E., Murphy, D. C., Krzeminski, W., et al. 1998, AJ, 116, 2475

Peletier, R. F., Davies, R. L., Illingworth, G. D., et al. 1990, AJ, 100, 1091

Prugniel, P., Nieto, J.-L., \& Simien, F. 1987, A\&A, 173, 49

Puzia, T. H., Kissler-Patig, M., Brodie, J. P., et al. 1999, AJ, 118, 2734

Rejkuba, M. 2001, A\&A, 369, 812

Renzini, A., \& Buzzoni, A. 1986, in Spectral evolution of galaxies, ed. C. Chiosi, \& A. Renzini (Dordrecht: Reidel), 135

Rhode, K. L., \& Zepf, S. E. 2001, AJ, 121, 210

Tonry, J. L., Blakeslee, J. P., Ajhar, E. A., \& Dressler, A. 2000, ApJ, 530,625

Whitmore, B. C., Sparks, W. B., Lucas, R. A., et al. 1995, ApJ, 545, L73

Worthey, G. 1994, ApJS, 95, 107

Zepf, S. E., \& Ashman, K. M. 1993, MNRAS, 264, 611

Zinn, R. 1985, ApJ, 293, 424 